\section{DMARDs schützen RA-Patienten vor Invalidität}

Mit Methotrexat und Biologika lassen sich bei Patienten mit rheumatoider Arthritis die Entzündungsvorgänge erfolgreich eindämmen. Langfristig hofft man, auf diese Weise die Gelenkfunktion der Patienten zu erhalten und sie vor Invalidität zu bewahren.

$\mathrm{E}_{\mathrm{n}}^{\mathrm{s}}$ gibt einen erfreulichen Trend: Immer weniger RA-Patienten werden aufgrund ihrer Erkrankung invalide. Die jährliche Rate sank bis Ende der 1990er-Jahre um $2 \%$, vermutlich dank der neueren Behandlungsstrategien mit Methotrexat (MTX). 1999 erweiterten die Biologika das therapeutische Repertoire. Welchen Einfluss die DMARDs und insbesondere die Biologika auf die langfristige Gelenkfunktion tatsächlich haben, überprüften US-amerikanische Rheumatologen in einer prospektiven Kohortenstudie.

Sie werteten die Daten von $4.651 \mathrm{~Pa}$ tienten aus, die in das Arthritis, Rheumatism and Aging Medical Information System (ARAMIS) eingespeist worden waren. Alle Patienten wurden zwischen 1983 und 2006 gelistet und anschließend halbjährlich deren Gelenkfunktion sowie der Schweregrad der Beeinträchtigung anhand des Health Assessment Questionnaire Disability Index (HAQ) bestimmt. Die Patienten sollten angeben, inwieweit sie ihre Krankheit beim Anziehen, der Körperpflege, beim Greifen, Aufstehen, Essen, Gehen und Putzen behindert sowie in ihrem Bewegungsradius und in ihrer Aktivität einschränkt. Der Grad konnte mit 0 bis 3 bewertet werden.

Die meisten Patienten waren weiblich (76\%), hatten eine weiße Hautfarbe (88\%) und waren im Durchschnitt seit 13 Jahren rheumakrank. Innerhalb der acht Beobachtungsjahre änderten sich die Behandlungsschemata: Immer mehr Patienten wurden mit MTX und später auch mit Biologika therapiert.

Im Untersuchungszeitraum verbesserten sich die HAQ-Scores der Patienten von 1,3 in den 1980er-Jahren, auf 1,2 in den 1990er-Jahre und weiter auf unter 1,1 in den 2000er-Jahren. Insgesamt ging die Aktivitätseinschränkung jährlich um 1,7\% zurück. Dabei ließ sich ein bestimmter zeitlicher Verlauf erkennen: Vor 1990, als die Behandlung noch aus- schließlich NSAR-basiert war, blieb der Grad der Einschränkung konstant hoch, erst in den 1990er-Jahren, als MTX auf den Plan kam und schließlich fast 45\% der Patienten mit dem DMARD behandelt wurden, verringerte sich die Beeinträchtigung deutlich $(\mathrm{p}=0,001)$. Dieser Trend setzte sich auch in den 2000erJahren mit Einführung der Biologika fort ( $\mathrm{p}<0,01)$, wenngleich nicht mehr so ausgeprägt. Dieses Ergebnis war unabhängig von Alter, Geschlecht, ethnischer Zugehörigkeit, Körpergewicht und dem Zeitpunkt der RA-Diagnose der Patienten.

Fazit: Mit Einführung von MTX und der Biologika in die RA-Therapie verbesserten sich jeweils die HAQ-Scores deutlich, so die Autoren. Die Patienten waren in ihrem täglichen Leben weniger eingeschränkt. Dieses Ergebnis unterstreiche die Bedeutung von MTX und der Biologika für die Behandlung der RA sowie die aktuell geltende Therapiestrategie, die Entzündungsvorgänge möglichst frühzeitig effektiv einzudämmen.

Dr. Dagmar Kraus

Krishnan E et al. Disability in rheumatoid arthritis in the era of biological treatments. Ann Rheum Dis 2012;71:213-218. doi:10.1136/annrheumdis-2011-200354

Kommentar: Die Ergebnisse dieser prospektiven Kohortenstudie von Krishnan et al. bestätigen in eindrucksvoller Weise den bereits aus anderen Studien bekannten positiven Effekt einer immunsuppressiven Therapie der RA auf den langfristigen Erhalt der Gelenkfunktion. Die bis in die 1980er-Jahre häufig praktizierte alleinige Therapie mit NSAR reicht hierfür nicht aus, erst mit einer regelmäßigen MTX-Therapie konnten hier eindeutige Fortschritte erreicht werden, die sich in der Ära der Biologika fortsetzen. Auch das in den vergangenen Jahren verbesserte Management anderer Risikofaktoren, beispielsweise der Rauchgewohnheiten von RA-Patienten,

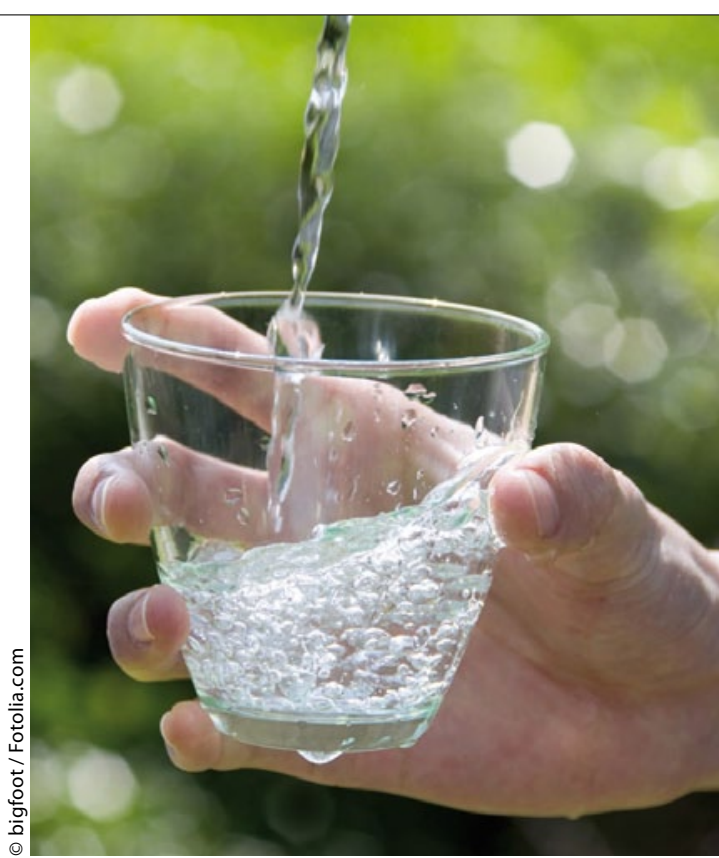

Alltägliche Handgriffe wie ein Glas zu halten sind schlecht behandelten Rheumapatienten oft nicht mehr möglich.

genügt nicht, um die gut dokumentierten und nachhaltigen Verbesserungen im HAQ-Score zu erklären. Dieser Fortschritt ist nachweislich auf eine frühzeitig einsetzende Therapie mit DMARDs oder mit Biologika zurückzuführen. Bemerkenswert ist in diesem Zusammenhang, dass selbst in Deutschland längst nicht alle infrage kommenden RA-Patienten eine rechtzeitige DMARD-Therapie erhalten und dass Biologika bei der RA in den USA weit häufiger verordnet werden als bei uns.

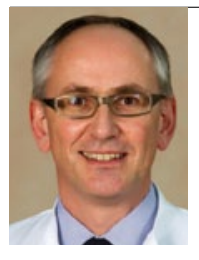

\section{Dr. Diethard Kaufmann} Chefarzt Rheumazentrum Oberammergau Klinik für Internistische Rheumatologie

Hubertusstr. 40, 82487 Oberammergau E-Mail: diethard.kaufmann@wz-kliniken.de Internet:

www.rheumazentrum-oberammergau.de www.wzk-portal.de 